\title{
A logarithmic Sobolev form of the Li-Yau parabolic inequality
}

\section{Dominique Bakry and Michel Ledoux}

\begin{abstract}
We present a finite dimensional version of the logarithmic Sobolev inequality for heat kernel measures of non-negatively curved diffusion operators that contains and improves upon the Li-Yau parabolic inequality. This new inequality is of interest already in Euclidean space for the standard Gaussian measure. The result may also be seen as an extended version of the semigroup commutation properties under curvature conditions. It may be applied to reach optimal Euclidean logarithmic Sobolev inequalities in this setting. Exponential Laplace differential inequalities through the Herbst argument furthermore yield diameter bounds and dimensional estimates on the heat kernel volume of balls.
\end{abstract}

\section{Introduction and main result}

The celebrated Li-Yau parabolic inequality [15] on a Riemannian manifold $M$ with dimension $n$ and non-negative Ricci curvature expresses that for any positive function $f$ on $M$ and any $t>0$,

$$
-\Delta\left(\log P_{t} f\right) \leq \frac{n}{2 t}
$$

(pointwise) where $\Delta$ is the Laplace-Beltrami operator on $M$ and

$$
P_{t}=\mathrm{e}^{t \Delta}
$$

the heat semigroup. This crucial bound has been widely used towards Harnack inequalities and refined heat kernel bounds (cf. [15], [9], [16], ...)

2000 Mathematics Subject Classification: 58J, 60H, 60J.

Keywords: Logarithmic Sobolev inequality, Li-Yau parabolic inequality, heat semigroup, gradient estimate, non-negative curvature, diameter bound. 
On the other hand, gradient estimates have been used in the context of abstract Markov diffusion operators to establish sharp functional inequalities for heat kernel measures. A convenient setting where this may be developed is presented in $[2,3]$. On some measure space $(E, \mathcal{E}, \mu)$, let $\mathrm{L}$ be a Markov generator associated to a semigroup $\left(P_{t}\right)_{t \geq 0}$, continuous in $\mathrm{L}^{2}(\mu)$, with invariant measure $\mu$. For a given nice algebra $\mathcal{A}$ of functions on $E$ in the $\mathrm{L}^{2}$-domain of $\mathrm{L}$, let $\Gamma$ be the symmetric non-negative bilinear operator on $\mathcal{A} \times \mathcal{A}$ defined by

$$
2 \Gamma(f, g)=\mathrm{L}(f g)-f \mathrm{~L} g-g \mathrm{~L} f, \quad f, g \in \mathcal{A} .
$$

The operator $\mathrm{L}$ is then said to be a diffusion if for every $C^{\infty}$ function $\Psi$ on $\mathbb{R}$ and every $f$ in $\mathcal{A}$,

$$
\mathrm{L} \Psi(f)=\Psi^{\prime}(f) \mathrm{L} f+\Psi^{\prime \prime}(f) \Gamma(f, f) .
$$

This hypothesis essentially expresses that L is a second order differential operator with no constant term and that we have a chain rule formula for $\Gamma, \Gamma(\Psi(f), g)=\Psi^{\prime}(f) \Gamma(f, g), f, g \in \mathcal{A}$.

One basic example is of course the Laplace-Beltrami operator $\Delta$ on a complete connected Riemannian manifold $M$ (with its Riemannian measure). For $\mathcal{A}$ the class, say, of $C_{c}^{\infty}$ functions on $M, \Gamma(f, f)$ is simply the Riemannian length (squared) $|\nabla f|^{2}$ of the gradient $\nabla f$ of $f \in \mathcal{A}$. The previous abstract framework includes a number of further examples of interest (cf. [2]). For example, one may consider $\mathrm{L}=\Delta+X$ where $X$ is a smooth vector field on $M$, or more general second order differential operators with no constant term. In particular, we deal below with the Ornstein-Uhlenbeck generator on $\mathbb{R}^{n}, \mathrm{~L} f(x)=\Delta f(x)-x \cdot \nabla f(x)$ with invariant measure the standard Gaussian measure $d \gamma_{n}(x)=(2 \pi)^{-n / 2} \mathrm{e}^{-|x|^{2} / 2} d x$. In all these explicit examples, it is easy to describe a nice algebra $\mathcal{A}$ of (bounded) functions on $E$ dense in the $\mathrm{L}^{2}$-domain of $\mathrm{L}$, stable by $\mathrm{L}$ and $P_{t}$, and by the action of $C^{\infty}$ functions. The stability by $P_{t}$ may not be satisfied even in basic examples such as non-degenerate second order differential operators with no constant term on a smooth (non-compact) manifold. This assumption is however not strictly necessary, and each example of interest has actually to be analyzed in its own. In order not to obscure the main ideas, we prefer to restrict to such a convenient framework.

Curvature, and dimension, in this setting are introduced via the $\Gamma_{2}$ operator defined by

$$
2 \Gamma_{2}(f, g)=\mathrm{L} \Gamma(f, g)-\Gamma(f, \mathrm{~L} g)-\Gamma(g, \mathrm{~L} f), \quad f, g \in \mathcal{A} .
$$


For simplicity, we write below $\Gamma f=\Gamma(f)=\Gamma(f, f)$ and similarly for $\Gamma_{2}$. In a Riemannian setting, Bochner's formula (cf. [8], [10]) indicates that

$$
\Gamma_{2}(f)=\Gamma_{2}(f, f)=\operatorname{Ric}(\nabla f, \nabla f)+\|\operatorname{Hess} f\|_{2}^{2}
$$

where Ric is the Ricci tensor on $M$ and $\|$ Hess $f \|_{2}$ is the Hilbert-Schmidt norm of the tensor of the second derivatives of $f$. We will say that $\mathrm{L}$ satisfies a curvature-dimension inequality $\operatorname{CD}(R, n)$ of curvature $R \in \mathbb{R}$ and dimension $0<n \leq \infty$ if, for all functions $f$ in $\mathcal{A}$ ( $\mu$-almost everywhere),

$$
\Gamma_{2}(f) \geq R \Gamma(f)+\frac{1}{n}(\mathrm{~L} f)^{2} .
$$

Clearly, if L satisfies a curvature-dimension inequality $\operatorname{CD}(R, n)$, then it also satisfies $\mathrm{CD}\left(R^{\prime}, n^{\prime}\right)$ for $R^{\prime} \leq R$ and $n^{\prime} \geq n$. A $n$-dimensional complete Riemannian manifold $(M, g)$ with Ricci curvature bounded below, or rather the Laplacian $\Delta$ on $M$, satisfies the inequality $\operatorname{CD}(R, n)$ with $R$ the infimum of the Ricci tensor over all unit tangent vectors and $n$ the topological dimension (since $\|$ Hess $f \|_{2}^{2} \geq \frac{1}{n}(\Delta f)^{2}$ ). If $\mathrm{L}=\Delta+\nabla h$ for a smooth function $h$, and if (and only if), as symmetric tensors,

$$
\nabla h \otimes \nabla h \leq(m-n)[\text { Ric }-\nabla \nabla h-\rho g]
$$

with $m \geq n$, then L satifies $\mathrm{CD}(\rho, m)$ (cf. [2, Proposition 6.2]. The dimension $n$ in (1.3) is not necessarily an integer, and reflects the analytic dimension of diffusion operators L (cf. [2] for further examples). The OrnsteinUhlenbeck operator is of curvature-dimension $\operatorname{CD}(1, \infty)$, but of no finite dimension whatsoever the dimension of the underlying state space.

One important aspect in this framework, at the starting point of this investigation, is that an infinite dimensional curvature condition $\operatorname{CD}(R, \infty)$ on the infinitesimal generator $\mathrm{L}$ may be translated equivalently on the associated semigroup $\left(P_{t}\right)_{t \geq 0}$. One classical such description is that $\Gamma_{2} \geq R \Gamma$, $R \in \mathbb{R}$, if and only if for every $f$ in $\mathcal{A}$ and every $t \geq 0$,

$$
\Gamma\left(P_{t} f\right) \leq \mathrm{e}^{-2 R t} P_{t}(\Gamma f)
$$

(This type of inequalities, here and below, are understood either everywhere or $\mu$-almost everywhere.) For further comparison, let us briefly recall the argument [3], [12]. Let, for $f \in \mathcal{A}$ and $t>0$ fixed, $\phi(s)=\mathrm{e}^{-2 R s} P_{s}\left(\Gamma\left(P_{t-s} f\right)\right)$, $0 \leq s \leq t$. Now, by definition of the $\Gamma_{2}$ operator,

$$
\phi^{\prime}(s)=2 \mathrm{e}^{-2 R s} P_{s}\left(\Gamma_{2}\left(P_{t-s} f\right)-R \Gamma\left(P_{t-s} f\right)\right) .
$$


Hence, by $\operatorname{CD}(R, \infty)$ applied to $P_{t-s} f$ for every $s, \phi$ is non-decreasing and (1.4) follows. For the converse, note that (1.4) is an equality at $t=0$ and

$$
0 \leq \lim _{t \rightarrow 0} \frac{1}{2 t}\left[\mathrm{e}^{-2 R t} P_{t}(\Gamma f)-\Gamma\left(P_{t} f\right)\right]=\Gamma_{2}(f)-R \Gamma(f) .
$$

If we let now

$$
\phi(s)=\mathrm{e}^{-2 R s} P_{s}\left(\frac{\left.\Gamma\left(P_{t-s} f\right)\right)}{P_{t-s} f}\right)=\mathrm{e}^{-2 R s} P_{s}\left(P_{t-s} f \Gamma\left(\log P_{t-s} f\right)\right), \quad 0 \leq s \leq t,
$$

for a strictly positive function $f$, then

$$
\left.\phi^{\prime}(s)=2 \mathrm{e}^{-2 R s} P_{s}\left(\frac{1}{g} \Gamma_{2}(g)-\frac{1}{g^{2}} \Gamma(g, \Gamma g)+\frac{1}{g^{3}} \Gamma^{2}(g)-R g \Gamma(\log g)\right]\right)
$$

where $g=P_{t-s} f$. The change of variable formula for the $\Gamma_{2}$ operator, consequence of the diffusion property (1.2) (cf. [2]), reads

$$
\Gamma_{2}(\log g)=\frac{1}{g^{2}} \Gamma_{2}(g)-\frac{1}{g^{3}} \Gamma(g, \Gamma g)+\frac{1}{g^{4}} \Gamma^{2}(g) .
$$

Therefore,

$$
\phi^{\prime}(s)=2 \mathrm{e}^{-2 R s} P_{s}\left(g\left[\Gamma_{2}(\log g)-R \Gamma(\log g)\right]\right) .
$$

Hence, again, under $\mathrm{CD}(R, \infty), \phi^{\prime}(s) \geq 0,0 \leq s \leq t$, and therefore

$$
P_{t} f \Gamma\left(\log P_{t} f\right) \leq \mathrm{e}^{-2 R t} P_{t}(f \Gamma(\log f)) .
$$

Applying (1.5) to $1+\varepsilon f$ and letting $\varepsilon \rightarrow 0$ recaptures (1.4) so that (1.5) is a further alternate characterization of the $\operatorname{CD}(R, \infty)$ condition. Observe also that when $R=0$, inequality (1.5) is equivalent by the diffusion property to

$$
P_{t} f \mathrm{~L}\left(\log P_{t} f\right) \geq P_{t}(f \mathrm{~L}(\log f))
$$

(for every $f \in \mathcal{A}$ and $t \geq 0$ ). These commutation properties are typically used in proofs of both spectral and logarithmic Sobolev inequalities for heat kernel measures. For example, if $\mathrm{L}$ satisfies $\operatorname{CD}(R, \infty)$ for some $R \in \mathbb{R}$, then for every $f$ in $\mathcal{A}$ and $t \geq 0$,

$$
d(t) \Gamma\left(P_{t} f\right) \leq P_{t}\left(f^{2}\right)-\left(P_{t} f\right)^{2} \leq c(t) P_{t}(\Gamma f)
$$

and

(1.8) $\frac{d(t)}{2} P_{t} f \Gamma\left(\log P_{t} f\right) \leq P_{t}(f \log f)-P_{t} f \log P_{t} f \leq \frac{c(t)}{2} P_{t}(f \Gamma(\log f))$ 
where

$$
c(t)=2 \int_{0}^{t} \mathrm{e}^{-2 R s} d s=\frac{1-\mathrm{e}^{-2 R t}}{R} \quad(=2 t \text { if } R=0)
$$

and

$$
d(t)=2 \int_{0}^{t} \mathrm{e}^{2 R s} d s=\frac{\mathrm{e}^{2 R t}-1}{R} \quad(=2 t \text { if } R=0) .
$$

For the proof of (1.7), write

$$
P_{t}\left(f^{2}\right)-\left(P_{t} f\right)^{2}=\int_{0}^{t} \frac{d}{d s} P_{s}\left(\left(P_{t-s} f\right)^{2}\right) d s=2 \int_{0}^{t} P_{s}\left(\Gamma\left(P_{t-s} f\right)\right) d s .
$$

Now, since $\phi(s)=\mathrm{e}^{-2 R s} P_{s}\left(\Gamma\left(P_{t-s} f\right)\right), 0 \leq s \leq t$, is non-decreasing, we get on the one hand

$$
P_{t}\left(f^{2}\right)-\left(P_{t} f\right)^{2} \leq 2 \int_{0}^{t} \mathrm{e}^{-2 R(t-s)} P_{t}(\Gamma f) d s=c(t) P_{t}(\Gamma f),
$$

while on the other

$$
P_{t}\left(f^{2}\right)-\left(P_{t} f\right)^{2} \geq 2 \int_{0}^{t} \mathrm{e}^{2 R(t-s)} \Gamma\left(P_{t} f\right) d s=d(t) \Gamma\left(P_{t} f\right) .
$$

For the proof of the logarithmic Sobolev inequalities (1.8), write for $f>0$,

$$
\begin{aligned}
P_{t}(f \log f)-P_{t} f \log P_{t} f & =\int_{0}^{t} \frac{d}{d s} P_{s}\left(P_{t-s} f \log P_{t-s} f\right) d s \\
& =\int_{0}^{t} P_{s}\left(P_{t-s} f \Gamma\left(\log P_{t-s} f\right)\right) d s
\end{aligned}
$$

and apply then in the same way that $\phi(s)=\mathrm{e}^{-2 R s} P_{s}\left(P_{t-s} f \Gamma\left(\log P_{t-s} f\right)\right)$, $0 \leq s \leq t$, is non-decreasing.

Arguing as for (1.4), but differentiating twice, shows that (1.7) and (1.8) holding for all $f$ and $t>0$ are also equivalent to the curvature condition $\mathrm{CD}(R, \infty)$. More refined isoperimetric inequalities are discussed in [5].

One crucial defect of the preceding approach is that it does not take into account the dimensional parameter $n$. For Laplace-Beltrami operators, conditions $\mathrm{CD}(R, n)$ and $\mathrm{CD}(R, \infty)$ are actually equivalent, but the dimensional parameter $n$ is then hidden in the very definition of the operator $L$. Therefore, the equivalent form of the Ricci lower bound given for example by (1.5), while well suited for infinite dimensional diffusions, is not accurate when one deals with finite dimensional problems (such as for example compactness problems in the Gromov-Hausdorff topology).

On the other hand, the Li-Yau inequality strongly relies on the finite dimensional property of the operator $\mathrm{L}$, but is not a priori equivalent with a lower bound on the Ricci curvature. 
One of the main observations of this work, that seemed to be have been overlooked, is that the semigroup procedure presented above may actually be performed similarly in finite dimension, and that the argument includes in this way the Li-Yau inequality (1.1). We thus describe, in a unified treatment, both functional inequalities for heat kernel measures and parabolic inequalities of finite dimensional Markov operators. The various inequalities take a simple form only when the curvature $R$ is zero, case on which we restrict ourselves henceforth. The main result of this paper is the following theorem.

Theorem 1. Let L be a diffusion operator. The following are equivalent:

(i) L satisfies a $C D(0, n)$ inequality;

(ii) For any positive function $f$ in $\mathcal{A}$ and any $t \geq 0$,

$$
P_{t} f \mathrm{~L}\left(\log P_{t} f\right) \geq P_{t}(f \mathrm{~L}(\log f))\left(1+\frac{2 t}{n} \mathrm{~L}\left(\log P_{t} f\right)\right)
$$

(iii) For any positive function $f$ in $\mathcal{A}$ and any $t \geq 0$,

$$
t \mathrm{~L} P_{t} f-\frac{n}{2} P_{t} f \log \left(1+\frac{2 t}{n} \mathrm{~L}\left(\log P_{t} f\right)\right) \leq P_{t}(f \log f)-P_{t} f \log P_{t} f
$$

(iv) For any positive function $f$ in $\mathcal{A}$ and any $t \geq 0$,

$$
\begin{aligned}
P_{t}(f \log f) & -P_{t} f \log P_{t} f \leq \\
& \leq t \mathrm{~L} P_{t} f+\frac{n}{2} P_{t} f \log \left(1-\frac{2 t}{n} \frac{P_{t}(f \mathrm{~L}(\log f))}{P_{t} f}\right) .
\end{aligned}
$$

The infinite dimensional case $n=\infty$ reduces to (1.6) and (1.8) (for $R=0$ ) respectively. Using that $\log (1+u) \leq u, u>-1$, Theorem 1 actually clearly shows how the new logarithmic Sobolev inequalities (1.10) and (1.11) improve upon (1.8) (when $R=0$ ).

As announced, Theorem 1 also encompasses the Li-Yau inequality (1.1). Actually, if we analyze (1.9), it turns out that whenever $P_{t} f \mathrm{~L}\left(\log P_{t} f\right)<0$, then (by (1.6)) $P_{t}(f \mathrm{~L}(\log f))<0$ so that necessarily

$$
1+\frac{2 t}{n} \mathrm{~L}\left(\log P_{t} f\right)>0
$$

which is the Li-Yau inequality in this context (it is obvious when $\mathrm{L}\left(\log P_{t} f\right)$ $\geq 0$ ). This inequality is implicit in the statement of the logarithmic Sobolev inequality (1.10) that may thus be considered as a logarithmic Sobolev form 
of the parabolic Li-Yau inequality. The new proof here may also be considered as a simplification over the original Li-Yau argument based on the maximum principle. Note that (1.9) (or (1.11)) also expresses, as a kind of converse, that

$$
\frac{P_{t}(f \mathrm{~L}(\log f))}{P_{t} f}<\frac{n}{2 t} .
$$

Proof of Theorem 1. We first show that (1.9), (1.10) or (1.11) imply the $\mathrm{CD}(0, n)$ hypothesis. Namely, if we apply (1.9), (1.10) and (1.11) to $1+\varepsilon f$ and let $\varepsilon \rightarrow 0$, we find respectively that

$$
\begin{gathered}
\Gamma\left(P_{t} f\right) \leq P_{t}(\Gamma f)-\frac{2 t}{n}\left(\mathrm{~L} P_{t} f\right)^{2}, \\
P_{t}\left(f^{2}\right)-\left(P_{t} f\right)^{2} \geq 2 t \Gamma\left(P_{t} f\right)+\frac{2 t^{2}}{n}\left(\mathrm{~L} P_{t} f\right)^{2},
\end{gathered}
$$

and

$$
P_{t}\left(f^{2}\right)-\left(P_{t} f\right)^{2} \leq 2 t P_{t}(\Gamma f)-\frac{2 t^{2}}{n}\left(\mathrm{~L} P_{t} f\right)^{2}
$$

As in infinite dimension, noticing that the first inequality (1.12) is an equality at $t=0$, we see that

$$
0 \leq \lim _{t \rightarrow 0} \frac{1}{2 t}\left[P_{t}(\Gamma f)-\Gamma\left(P_{t} f\right)-\frac{2 t}{n}\left(\mathrm{~L} P_{t} f\right)^{2}\right]=\Gamma_{2}(f)-\frac{1}{n}(\mathrm{~L} f)^{2},
$$

and therefore the $\operatorname{CD}(0, n)$ condition holds. Differentiating twice yields the same conclusion for (1.13) or (1.14). This shows that any of the inequalities (1.9), (1.10) or (1.11) imply the curvature-dimension $\operatorname{CD}(0, n)$ hypothesis. The theorem establishes that (1.12), (1.13) and (1.14) are also equivalent forms of the $\operatorname{CD}(0, n)$ inequality.

Let us turn to the converse and assume that $n<\infty$. Start with the proof of (1.9) that follows the one of (1.5). Given $f>0$ on $\mathcal{A}$, if

$$
\phi(s)=P_{s}\left(P_{t-s} f \Gamma\left(\log P_{t-s} f\right)\right), \quad 0 \leq s \leq t,
$$

we have seen that

$$
\left.\phi^{\prime}(s)=2 P_{s}\left(P_{t-s} f \Gamma_{2}\left(\log P_{t-s} f\right)\right]\right) .
$$

Hence, under the $\operatorname{CD}(0, n)$ condition,

$$
\phi^{\prime}(s) \geq \frac{2}{n} P_{s}\left(g[\mathrm{~L}(\log g)]^{2}\right)
$$


where we set $g=P_{t-s} f$ as before. Since

$$
g[\mathrm{~L}(\log g)]^{2}=\frac{1}{g}\left[\mathrm{~L} g-\frac{\Gamma g}{g}\right]^{2},
$$

by the Cauchy-Schwarz inequality in the form of

$$
P_{s}\left(\frac{A^{2}}{g}\right) \geq \frac{P_{s}(A)^{2}}{P_{s}(g)}
$$

it follows that

$$
\phi^{\prime}(s) \geq \frac{2}{n P_{t} f}\left[\phi(s)-\mathrm{L} P_{t} f\right]^{2}, \quad 0 \leq s \leq t .
$$

In other words,

$$
-\left(\frac{1}{\phi-\mathrm{L} P_{t} f}\right)^{\prime} \geq \alpha
$$

on $[0, t]$, where we set for simplicity $\alpha=\frac{2}{n P_{t} f}>0$. We then only need to integrate between 0 and $t$ to get that, for every $0 \leq u<v \leq t$,

$$
\frac{1}{\phi(u)-\mathrm{L} P_{t} f}-\frac{1}{\phi(v)-\mathrm{L} P_{t} f} \geq \alpha(v-u) .
$$

Since $\phi$ is non-decreasing,

$$
\left[\phi(v)-\mathrm{L} P_{t} f\right]-\left[\phi(u)-\mathrm{L} P_{t} f\right] \geq \alpha(v-u)\left[\phi(u)-\mathrm{L} P_{t} f\right]\left[\phi(v)-\mathrm{L} P_{t} f\right]
$$

whatsoever the signs of $\phi(u)-\mathrm{L} P_{t} f$ and $\phi(v)-\mathrm{L} P_{t} f$. Since $\phi(0)-\mathrm{L} P_{t} f=$ $-P_{t} f \mathrm{~L}\left(\log P_{t} f\right)$ and $\phi(t)-\mathrm{L} P_{t} f=-P_{t}(f \mathrm{~L}(\log f))$, the preceding with $u=0$ and $v=t$ already yields (1.9).

To reach the logarithmic Sobolev inequalities (1.10) and (1.11), write as above

$$
P_{t}(f \log f)-P_{t} f \log P_{t} f=\int_{0}^{t} \phi(s) d s .
$$

We derive from (1.16) that for every $0 \leq s \leq t$,

$$
\frac{1}{\alpha s-\left[\phi(0)-\mathrm{L} P_{t} f\right]^{-1}} \leq \phi(s)-\mathrm{L} P_{t} f \leq \frac{1}{\alpha(t-s)-\left[\phi(t)-\mathrm{L} P_{t} f\right]^{-1}}
$$

Actually, the left-hand side follows from (1.16) when $\phi(0)>\operatorname{L} P_{t} f$, and is obvious when both $\phi(0) \leq \mathrm{L} P_{t} f$ and $\phi(s) \leq \mathrm{L} P_{t} f$ since $\phi$ is non-decreasing. Similarly for the right-hand side. Integrating from 0 to $t$ then yields the two inequalities (1.10) and (1.11). The proof of the theorem is complete. 
While some statements analogous to Theorem 1 may be produced under a curvature-dimension condition $\mathrm{CD}(R, n)$ with $R \neq 0$, it rapidly appears that the corresponding conclusions are much less appealing, as it is also the case with the classical Li-Yau inequality [15]. Indeed, the differential inequality (1.15) on

$$
\phi(s)=P_{s}\left(P_{t-s} f \Gamma\left(\log P_{t-s} f\right)\right), \quad 0 \leq s \leq t,
$$

now takes the form

$$
\phi^{\prime}(s) \geq \frac{2}{n P_{t} f}\left[\phi(s)-\mathrm{L} P_{t} f\right]^{2}+2 R \phi(s) .
$$

Solutions of the differential inequality heavily depend on the initial condition. (In case $R=0$, the Li-Yau inequality is just the fact that this differential inequality does not explode on $[0, t]$.) Let us briefly discuss the resulting conclusion in case $R>0$, and for simplicity only at the level of the Li-Yau estimates. As a result, when $4 \mathrm{~L} P_{t} f / n R P_{t} f \geq 1$,

$$
-\mathrm{L}\left(\log P_{t} f\right) \leq \frac{n R}{2}[Z \operatorname{cotan}(t R Z)-1]
$$

where $Z=\left|\left(4 \mathrm{~L} P_{t} f / n R P_{t} f\right)-1\right|^{1 / 2}$. When $4 \mathrm{~L} P_{t} f / n R P_{t} f \leq 1$, replace cotan by coth.

Inequality (1.17) may be used towards useful semigroup bounds under positive curvature. Observe namely that $-\mathrm{L}\left(\log P_{t} f\right) \geq-\mathrm{L} P_{t} f / P_{t} f$. Then, in case $4 \mathrm{~L} P_{t} f / n R P_{t} f \geq 1$, it follows in particular from (1.17) that

$$
\frac{1}{2}\left(\frac{1}{Z}-Z\right) \leq \operatorname{cotan}(t R Z)
$$

or in other words,

$$
R t \leq \frac{1}{Z}\left(\frac{\pi}{2}-\arctan \left(\frac{1}{2}\left(\frac{1}{Z}-Z\right)\right) .\right.
$$

The map

$$
x \mapsto \frac{1}{x}\left(\frac{\pi}{2}-\arctan \left(\frac{1}{x}-x\right)\right)
$$

is non-increasing on $(0, \infty)$, and converges to 2 at $x=0$. Hence, in this case, $R t \leq 2$. As a conclusion,

$$
\partial_{t}\left(\log P_{t} f\right) \leq \frac{n R}{4}
$$

as soon as $t \geq 2 / R$. This bound has been put forward first in [6] by a completely different method (the maximum principle as for the classical LiYau inequality). 
Consider now the case $4 \mathrm{~L} P_{t} f / n R P_{t} f \leq 1$ that thus occurs as soon as $t$ is large enough. We then get that

$$
\frac{1}{2}\left(Z+\frac{1}{Z}\right) \leq \operatorname{coth}(R t Z)
$$

or in other words,

$$
R t \leq \frac{1}{Z} \log \left(\frac{1+Z}{|Z-1|}\right)
$$

The map $H: x \mapsto \frac{1}{x} \log \left(\frac{1+x}{|x-1|}\right)$ increases from 2 to $+\infty$ on $(0,1)$, and decreases from $+\infty$ to 0 on $(1, \infty)$. It follows that $Z$ is bounded from below by $K(R t)$ where $K$ is the inverse map of $H$ on $(0, \infty)$. As $x \rightarrow \infty$, $K(x)=1-2 \mathrm{e}^{-x}\left(1+O\left(x \mathrm{e}^{-x}\right)\right)$ so that

$$
\partial_{t}\left(\log P_{t} f\right) \leq \frac{n R}{4}\left(1-K^{2}(R t)\right)
$$

This leads to an upper bound on the heat semigroup of the form

$$
P_{t} f \leq \exp \left(n \mathrm{e}^{-R t}(1+\varepsilon(t))\right)
$$

as $t \rightarrow \infty$ for positive functions $f$ such that $\int f d \mu=1$. Lower bounds may be obtained similarly from the upper bound on $Z$ by the inverse function of $H$ on the interval $(1, \infty)$. (When $R=0$, the Li-Yau inequality actually implies that $t^{n / 2} P_{t} f$ is non-decreasing.)

The extension of Theorem 1 to a $\operatorname{CD}(R, n), R \neq 0$, hypothesis thus takes a somewhat intricate form. However, it might be worthwhile mentioning that among the heat kernel inequalities equivalent to $\mathrm{CD}(0, n),(1.12),(1.13)$ and (1.14) may easily be adapted under a $\operatorname{CD}(R, n), R \neq 0$, condition. Arguing indeed as for (1.4) and (1.7) shows that the $\operatorname{CD}(R, n)$ curvature-dimension assumption is equivalent to either

$$
\Gamma\left(P_{t} f\right) \leq \mathrm{e}^{-2 R t} P_{t}(\Gamma f)-\frac{c(t)}{n}\left(\mathrm{~L} P_{t} f\right)^{2}
$$

or

$d(t) \Gamma\left(P_{t}\right)+\frac{2 D(t)}{n}\left(\mathrm{~L} P_{t} f\right)^{2} \leq P_{t}\left(f^{2}\right)-\left(P_{t} f\right)^{2} \leq c(t) P_{t}(\Gamma f)-\frac{2 C(t)}{n}\left(\mathrm{~L} P_{t} f\right)^{2}$,

$t \geq 0, f \in \mathcal{A}$, where we recall that $c(t)=\frac{1-\mathrm{e}^{-2 R t}}{R}$ and $d(t)=\frac{\mathrm{e}^{2 R t}-1}{R}(c(t)=$ $d(t)=2 t$ if $R=0)$, and where $C(t)=\int_{0}^{t} c(s) d s$ and $D(t)=\int_{0}^{t} d(s) d s, t \geq 0$. 
The rest of the paper will be devoted to several consequences of the main Theorem 1. As a first by-product, we show in Section 2 that under the finite dimensional $\mathrm{CD}(0, n)$ hypothesis, and provided that for any function $f$ in $\mathcal{A}$,

$$
\lim _{t \rightarrow \infty}(4 \pi t)^{n / 2} P_{t}(f)=\int f d \mu
$$

then the optimal logarithmic Sobolev inequality of $\mathbb{R}^{n}$ holds, namely, for all $f>0$ with $\int f d \mu=1$,

$$
\int f \log f d \mu \leq \frac{n}{2} \log \left(\frac{1}{2 n \pi \mathrm{e}} \int \frac{\Gamma(f)}{f} d \mu\right)
$$

We actually discuss how this logarithmic Sobolev inequality is related to the one of Theorem 1 for the heat kernel on $\mathbb{R}^{n}$, and thus the standard Gaussian measure. In particular, we emphasize a new finite dimensional version of the standard logarithmic Sobolev inequality for Gaussian measures, of interest in its own. In the last section, we discuss exponential bounds on Laplace transforms of Lipschitz functions that are drawn from the improved logarithmic Sobolev inequalities by the Herbst argument. With this tool, we investigate dimensional lower estimates on heat kernel measures of balls as well as bounds on the diameter when the underlying measure $\mu$ is finite. The important aspect is that these bounds will reflect, in accordance with the Euclidean case, the underlying finite dimension.

\section{Optimal Euclidean logarithmic Sobolev inequalities}

The optimal logarithmic Sobolev inequality in $\mathbb{R}^{n}$ states that for any smooth positive function $f$ on $\mathbb{R}^{n}$ with $\int f d x=1$,

$$
\int f \log f d x \leq \frac{n}{2} \log \left(\frac{1}{2 n \pi \mathrm{e}} \int \frac{|\nabla f|^{2}}{f} d x\right)
$$

After a change of functions, this inequality is actually equivalent to the logarithmic Sobolev inequality for Gaussian measures, and has been first put forward in this way in [7]. Start indeed from the Gross logarithmic Sobolev inequality [11] for the standard Gaussian measure $\gamma_{n}$ on $\mathbb{R}^{n}$ with density $\varphi_{n}(x)=(2 \pi)^{-n / 2} \exp \left(-|x|^{2} / 2\right)$ with respect to Lebesgue measure, which indicates that, for every smooth positive function $g$ on $\mathbb{R}^{n}$ with $\int g d \gamma_{n}=1$,

$$
\int g \log g d \gamma_{n} \leq \frac{1}{2} \int \frac{|\nabla g|^{2}}{g} d \gamma_{n}
$$


Set $f=\varphi_{n} g$ so that $\int f d x=1$. Then

$$
\int f \log \left(f(2 \pi)^{n / 2} \mathrm{e}^{|x|^{2} / 2}\right) d x \leq \frac{1}{2} \int \frac{1}{f}|\nabla f+x f|^{2} d x .
$$

An integration by parts easily yields

$$
\int f \log f d x \leq \frac{1}{2} \int \frac{|\nabla f|^{2}}{f} d x-\frac{n}{2} \log (2 \pi)-n .
$$

Changing $f$ into $\sigma^{n} f(\sigma x), \sigma>0$, which still satisfies the normalization $\int f d x=1$, shows that, for every $\sigma>0$ thus,

$$
\int f \log f d x \leq \frac{\sigma^{2}}{2} \int \frac{|\nabla f|^{2}}{f} d x-\frac{n}{2} \log (2 \pi)-n-n \log \sigma .
$$

Optimizing in $\sigma>0$ then yields (2.1). Since we started from the logarithmic Sobolev inequality for $\gamma_{n}$ with its best constant for which exponential functions are extremal, the constant in (2.1) is optimal, and Gaussian functions saturate the inequality.

Inequality (2.1) is closely related to the logarithmic Sobolev inequality of Theorem 1. Rather surprisingly, it turns out (and this is actually the way we discovered Theorem 1) that if we perform back a change of functions from (2.1) with the Gaussian extremals, we end up with the logarithmic Sobolev inequality of Theorem 1. Indeed, applying (2.1) to $f(y)=g(y) p_{t}(x, y)$ where

$$
p_{t}(x, y)=\frac{1}{(4 \pi t)^{n / 2}} \exp \left(-\frac{|x-y|^{2}}{4 t}\right)
$$

and proceeding as above now yields (1.11) for the heat kernel measure $P_{t}(d y)=p_{t}(x, y) d y$. If we read this inequality at time $t=\frac{1}{2}$, we obtain the following finite dimensional improved version of the Gross logarithmic Sobolev inequality (2.2) for the Gaussian measure $\gamma_{n}$ on $\mathbb{R}^{n}$.

Proposition 2. Let

$$
d \gamma_{n}(x)=(2 \pi)^{-n / 2} \exp \left(-|x|^{2} / 2\right) d x
$$

be the standard Gaussian measure on $\mathbb{R}^{n}$. For any smooth positive function $g$ on $\mathbb{R}^{n}$ such that $\int g d \gamma_{n}=1$,

$$
\int g \log g d \gamma_{n} \leq \frac{1}{2} \int \Delta g d \gamma_{n}+\frac{n}{2} \log \left(1-\frac{1}{n} \int g \Delta(\log g) d \gamma_{n}\right)
$$


Extremal functions are given here by the Gaussian kernel. Using that $\log (1+u) \leq u, u>-1$, we recover the classical form of the logarithmic Sobolev inequality for $\gamma_{n}$. As for (1.10), note that the logarithmic Sobolev inequality of Proposition 2 contains implicitely that

$$
1-\frac{1}{n} \int g \Delta(\log g) d \gamma_{n}>0
$$

It might worthwhile mentioning further that if we rewrite the spectral gap inequality (1.14) for $\gamma_{n}$ (equivalently, apply Proposition 2 to $1+\varepsilon g$ and let $\varepsilon \rightarrow 0$ ), we get

$$
\int g^{2} d \gamma_{n}-\left(\int g d \gamma_{n}\right)^{2} \leq \int|\nabla g|^{2} d \mu-\frac{1}{2 n}\left(\int \Delta g d \gamma_{n}\right)^{2} .
$$

The meaning of this inequality is somewhat strange. By integration by parts,

$$
\int \Delta g d \gamma_{n}=\int x \cdot \nabla g d \gamma_{n}=-\int g \mathrm{~L}\left(|x|^{2}\right) d \gamma_{n}
$$

where $\mathrm{L}=\Delta-x \cdot \nabla$ is the Ornstein-Uhlenbeck generator with invariant measure $\gamma_{n}$. Now $|x|^{2}-n$ is an eigenfunction of $-\mathrm{L}$ with eigenvalue 2, $-\mathrm{L}\left(|x|^{2}-n\right)=2\left(|x|^{2}-n\right)$. Applied to a mean zero function $g=g_{2}+G$ where $g_{2}=C\left(|x|^{2}-n\right)(C \neq 0)$ and $G$ is orthogonal to $g_{2},(2.3)$ amounts to

$$
\int G^{2} d \gamma_{n} \leq \int g_{2}^{2} d \gamma_{n}+\int|\nabla G|^{2} d \gamma_{n}-\frac{1}{2 n}\left(\int g_{2}\left(|x|^{2}-n\right) d \gamma_{n}\right)^{2} .
$$

Since $\int\left(|x|^{2}-n\right)^{2} d \gamma_{n}=2 n$, we see that (2.3) simply follows from the spectral gap inequality $\int G^{2} d \gamma_{n} \leq \int|\nabla G|^{2} d \gamma_{n}$ applied to $G$.

As a consequence of Theorem 1, we state next the logarithmic Sobolev inequality of the type (2.1), with the optimal Euclidean constant, for the invariant measure $\mu$ under a $\mathrm{CD}(0, n)$ condition.

Theorem 3. Let $\mathrm{L}$ be a diffusion operator with curvature-dimension $\mathrm{CD}(0, n)$, $n<\infty$. Assume that for all functions $f$,

$$
\lim _{t \rightarrow \infty}(4 \pi t)^{n / 2} t \mathrm{~L} P_{t} f=-\frac{n}{2} \int f d \mu
$$

(almost everywhere). Then, for every $f>0$ with $\int f d \mu=1$,

$$
\int f \log f d \mu \leq \frac{n}{2} \log \left(\frac{1}{2 n \pi \mathrm{e}} \int \frac{\Gamma(f)}{f} d \mu\right) .
$$


It is immediate from $(2.4)$ that for every $f$ (in $\mathcal{A})$,

$$
\lim _{t \rightarrow \infty}(4 \pi t)^{n / 2} P_{t}(f)=\int f d \mu
$$

(almost everywhere). The proof of Theorem 3 is then an immediate consequence of Theorem 1 by letting $t \rightarrow \infty$. Conversely, (2.5) implies (2.4) along a subsequence, which is enough for the proof of Theorem 3. This theorem thus answers a question left open in [4]. In a Riemannian manifold $M$ with dimension $n$ and non-negative Ricci curvature, it follows from the work of P. Li [14] that whenever (2.5) holds, then $M$ is isometric to $\mathbb{R}^{n}$, and thus Theorem 3 holds.

The main interest in Theorem 3 however lies in more general diffusion operators L than Laplacian on Riemannian manifolds. In particular, the logarithmic Sobolev inequality (with optimal Euclidean constants) of Theorem 3 has been used in [4] to derive optimal (Euclidean) heat kernel bounds in the form of $\left\|P_{t} f\right\|_{\infty} \leq(4 \pi t)^{-n / 2}\|f\|_{1}, t>0$.

It should be observed furthermore that the form of the Gaussian logarithmic Sobolev inequality given by Proposition 2 is equivalent in Euclidean space to the $\operatorname{CD}(0, n)$ condition through dilations and translations. There are other functional inequalities in Euclidean spaces which share the same property: For example, a modified form of the spectral gap inequality on the sphere read through stereographic projection (see [17]). It would be worthwhile to study which are the functional inequalities with this property. Neither the classical Euclidean Sobolev inequality, nor the spherical Sobolev inequality (through stereographic projection) share this property.

\section{Exponential integrability and diameter bounds}

In this final section, we exploit the logarithmic Sobolev inequalities (1.11) of Theorem 1 towards bounds on the Laplace transform of Lipschitz functions by the Herbst argument. We namely take advantage of the improved finite dimensional logarithmic Sobolev inequalities to produce quantitative estimates reflecting the underlying dimension. These bounds may be used to estimate heat kernel measures of balls in accordance with the Euclidean example. They may also be used for diameter bounds when the underlying measure $\mu$ is finite.

To this task, let $f$ be a (bounded) function in $\mathcal{A}$ such that $\Gamma(f) \leq 1$, and set

$$
\psi(\lambda, t)=\frac{1}{\lambda} \log P_{t}\left(\mathrm{e}^{\lambda f}\right), \quad \lambda \in \mathbb{R}, t \geq 0
$$

(with the convention that $\left.\psi(0, t)=P_{t} f\right)$. 
Applying the logarithmic Sobolev inequality (1.8) to $\mathrm{e}^{\lambda f}, \lambda \in \mathbb{R}$, the classical Herbst argument [1], [13] shows that, for every fixed $t$,

$$
\partial_{\lambda} \psi \leq \frac{c(t)}{2}
$$

so that

$$
\psi(\lambda, t) \leq \lambda P_{t} f+\frac{1}{2} c(t) \lambda, \quad \lambda \in \mathbb{R},
$$

$(c(t)=2 t$ when $R=0)$.

Here, we make use of the improved, finite dimensional, logarithmic Sobolev inequality (1.11) by further introducing a partial derivative in time. In the context thus of Theorem 1 , under a finite dimensional $\operatorname{CD}(0, n)$ hypothesis, inequality (1.11) applied to $\mathrm{e}^{\lambda f}$ now shows that, for every $\lambda>0, t>0$,

$$
\lambda \partial_{\lambda} \psi-t \partial_{t} \psi \leq \frac{n}{2 \lambda} \log \left(1+\frac{2 \lambda t}{n}\left[\lambda-\partial_{t} \psi\right]\right) .
$$

To analyze this differential inequality, set $t=(a \lambda+b)^{-1}$, and bound above $\log u$ by $\rho u-\log \rho-1$ at $\rho=b(a \lambda+b)^{-1}>0$, so that the function

$$
\psi\left(\frac{1}{a \lambda+b}, \lambda\right)+\frac{n}{2 \lambda} \log \left(\frac{a \lambda+b}{b}\right)+\frac{b}{a(a \lambda+b)}
$$

is non-increasing for all choices of the parameters $a$ and $b$ as long as $a \lambda+b>0$. In other words, along the curves $t=(a \lambda+b)^{-1}$,

$$
\psi-\frac{n}{2 \lambda} \log (b t)+\frac{b t}{a}
$$

is non-increasing. Under the conditions $\lambda_{2}>\lambda_{1}>0$ and $\lambda_{2} t_{2}>\lambda_{1} t_{1}>0$, we may take

$$
b=\frac{\lambda_{2} t_{2}-\lambda_{1} t_{1}}{t_{1} t_{2}\left(\lambda_{2}-\lambda_{1}\right)} \quad \text { and } \quad \frac{b}{a}=\frac{\lambda_{2} t_{2}-\lambda_{1} t_{1}}{t_{1}-t_{2}} .
$$

We then get

$$
\begin{aligned}
& \psi\left(\lambda_{2}, t_{2}\right)-\psi\left(\lambda_{1}, t_{1}\right) \\
& \quad \leq \lambda_{2} t_{2}-\lambda_{1} t_{1}+\frac{n}{2 \lambda_{1} \lambda_{2}}\left(\lambda_{2} \log t_{2}-\lambda_{1} \log t_{1}-\left(\lambda_{2}-\lambda_{1}\right) \log \left(\frac{\lambda_{2} t_{2}-\lambda_{1} t_{1}}{\lambda_{2}-\lambda_{1}}\right)\right) .
\end{aligned}
$$

This general inequality may be used to produce various bounds of interest on the Laplace transform of $f$ under $P_{t}$. For example, letting $\lambda_{1} \rightarrow 0$, we get, for every $\lambda>0$,

$$
\psi\left(\lambda, t_{2}\right) \leq P_{t_{1}} f+\lambda t_{2}+\frac{n}{2 \lambda}\left(\log \left(\frac{t_{2}}{t_{1}}\right)+\frac{t_{1}}{t_{2}}-1\right) .
$$


Alternatively, letting $\lambda_{1} \rightarrow \lambda_{2}=\lambda$, one also gets

$$
\psi\left(\lambda, t_{2}\right) \leq \psi\left(\lambda, t_{1}\right)+\lambda\left(t_{2}-t_{1}\right)+\frac{n}{2 \lambda} \log \left(\frac{t_{2}}{t_{1}}\right) .
$$

In another direction, one may fix $\left(\lambda_{1}, t_{1}\right)$ and set $\lambda_{2} t_{2}=a>\lambda_{1} t_{1}$, and let then $t_{2} \rightarrow 0$. Using Jensen's inequality $\psi(\lambda, t) \geq P_{t} f$, we get in the limit a bound on $f$ of the form

$$
f \leq \psi\left(\lambda_{1}, t_{1}\right)+a-\lambda_{1} t_{1}+\frac{n}{2 \lambda_{1}} \log \left(\frac{a}{a-\lambda_{1} t_{1}}\right) .
$$

The optimal bound is achieved by the choice of

$$
a=\frac{\lambda_{1} t_{1}}{2}\left(1+\sqrt{1+\frac{2 n}{\lambda_{1}^{2} t_{1}}}\right) .
$$

We now briefly sketch how to produce from the preceding Laplace inequalities, sharp dimensionl bounds on heat kernel measures of balls. Starting from (3.2) with the optimal choice of $a$ given by (3.3), we have that

$$
f \leq \psi(\lambda, t)+\frac{1}{\lambda} G\left(\frac{1}{\lambda^{2} t}\right)
$$

where

$$
G(x)=\frac{1}{2 x}(\sqrt{1+2 n x}-1)-\frac{n}{2} \log \left(1-\frac{2}{\sqrt{1+2 n x}+1}\right) .
$$

Now, $G(x) \sim-\frac{n}{2} \log x$ as $x \rightarrow 0$ while $G(x) \sim \sqrt{\frac{2 n}{x}}$ as $x \rightarrow \infty$. More precisely, the Taylor expansion of $G$ at $x=0$ yields

$$
G(x)=-\frac{n}{2} \log \left(\frac{n x}{2 \mathrm{e}}\right)+O(x) .
$$

For simplicity, denote by $C$ the best constant such that $G(x) \leq-\frac{n}{2} \log x+C$ for $x \in(0,1]$. Take then $f=-d\left(x_{0}, x\right)$ where $x_{0}$ is a fixed point in $E$, and let $B$ be the ball $B=\left\{x ; d\left(x, x_{0}\right) \leq r\right\}$. Since

$$
\mathrm{e}^{\lambda f} \leq \mathrm{e}^{-\lambda r} \mathbf{1}_{B^{c}}+\mathbf{1}_{B}
$$

it follows that

$$
\mathrm{e}^{\lambda \psi(\lambda, t)}=P_{t}\left(\mathrm{e}^{\lambda f}\right) \leq \mathrm{e}^{-\lambda r}+P_{t}\left(\mathbf{1}_{B}\right)\left(x_{0}\right) .
$$

We thus draw the lower bound

$$
P_{t}\left(\mathbf{1}_{B}\right)\left(x_{0}\right) \geq \exp \left(-G\left(\frac{1}{\lambda^{2} t}\right)\right)-\mathrm{e}^{-\lambda r} .
$$


For example, setting $\lambda=\frac{1}{r}$, so far as $r \leq \sqrt{t}$, we get that

$$
P_{t}\left(\mathbf{1}_{B}\right)\left(x_{0}\right) \geq \mathrm{e}^{-C}\left(\frac{r}{\sqrt{t}}\right)^{n}-\mathrm{e}^{-1} .
$$

In the range $r \sim \sqrt{t}$, this lower bound may of course be compared with the Euclidean case for which

$$
P_{t}\left(\mathbf{1}_{B}\right)\left(x_{0}\right) \geq \mathrm{e}^{-1 / 4} \frac{\omega_{n}}{(4 \pi)^{n / 2}}\left(\frac{r}{\sqrt{t}}\right)^{n}, \quad r \leq \sqrt{t},
$$

where $\omega_{n}$ is the volume of the unit ball in $\mathbb{R}^{n}$. We did not investigate carefully optimal constants in (3.4).

In the last part, we turn to diameter bounds. To this task, assume for what follows that $\mu$ is finite, normalized to be a probability measure. If we let $\lambda_{1} \rightarrow 0$ with $t_{1}=t \geq 0$ fixed in (3.2), we get in the limit that, for every $f$ with $\Gamma(f) \leq 1$,

$$
f \leq P_{t} f+\sqrt{2 n t}
$$

(Such inequalities appear implicity in earlier contributions, such as for example [18]).

A typical choice for the function $f$ is the distance function. To this task, assume that we may speak of the distance between $x$ and $y$ in $E$ defined by

$$
d(x, y)=\sup \{h(x)-h(y) ; \Gamma(h) \leq 1\} .
$$

Let $D=D(\mathrm{~L})$ be the diameter of $\mathrm{L}$ (relative to $\mathcal{A}$ ). As a consequence of the preceding, we will conclude that under a finite dimensional $\operatorname{CD}(0, n)$ hypothesis and a finite invariant measure, the diameter is bounded as soon as the distance function is integrable. The conclusion applies in particular to Riemannian manifolds with non-negative Ricci curvature and finite volume (we are not aware of a reference where such a result appears).

Proposition 4. Let L be a diffusion operator with curvature-dimension $C D(0, n), n<\infty$, and finite (normalized) invariant measure $\mu$. Then, if

$$
\iint d(x, y) d \mu(x) d \mu(y)<\infty
$$

we have $D=D(\mathrm{~L})<\infty$. Moreover,

$$
D \leq C_{n} \iint d(x, y) d \mu(x) d \mu(y)
$$

where $C_{n}$ only depends on $n$. 
The integrability condition on the distance function in Proposition 4 is of course equivalent to saying that $\int d\left(x, x_{0}\right) d \mu(x)<\infty$ for some, or all, $x_{0} \in E$.

Proof. The proof makes use of the Harnack inequality

$$
P_{t} f(x) \leq 2^{n} P_{2 t} f(y) \mathrm{e}^{d(x, y)^{2} / 4 t},
$$

$f \geq 0, t>0, x, y \in E$, consequence of the Li-Yau inequality, cf. [15, 9, 16] (and we henceforth assume the required setting for this to apply). Assume furthermore that $\Gamma(f) \leq 1$. Then, integrating the Harnack inequality (3.6) with respect to $d \mu(y)$ on the ball $B(x, r)$ with center $x$ and radius $r>0$, it follows together with (3.5) that, for every $x \in E, r, t>0$,

$$
\mu(B(x, r)) f(x) \leq 2^{n} \mathrm{e}^{r^{2} / 4 t} \int f d \mu+\sqrt{2 n t} .
$$

We proceed in two steps and first show that when $d$ is integrable,

$$
Z=\sup _{x \in E} \int d(x, z) d \mu(z)<\infty
$$

To this aim, apply (3.7) to the 1-Lipschitz function

$$
f(x)=\int d(x, z) d \mu(z)
$$

to get

$$
\mu(B(x, r)) \int d(x, z) d \mu(z) \leq 2^{n} \mathrm{e}^{r^{2} / 4 t} \iint d(y, z) d \mu(y) d \mu(z)+\sqrt{2 n t} .
$$

Using that

$$
\mu(B(x, r)) \geq 1-\frac{1}{r} \int d(x, z) d \mu(z)
$$

and letting $r=2 \int d(x, z) d \mu(z)$ and $t=r^{2} / 128 n$ proves that

$$
\int d(x, z) d \mu(z) \leq 2^{n+2} \mathrm{e}^{32 n} \iint d(y, z) d \mu(y) d \mu(z) .
$$

Since $x$ is arbitrary, (3.8) follows.

In the second step, apply $(3.7)$ to $f(x)=d(x, z)$ for every $z \in E$ to get that, for every $x, z \in E, r, t>0$,

$$
\mu(B(x, r)) d(x, z) \leq 2^{n} \mathrm{e}^{r^{2} / 4 t} Z+\sqrt{2 n t} .
$$


It already follows that the diameter of $E$ is finite. Indeed, $x$ being fixed, choose $r=r(x)$ such that, for example, $\mu(B(x, r)) \geq \frac{1}{2}$ so that $d(x, z) \leq$ $C(x)<\infty$ for every $z$. Hence $D<\infty$. Apply then (3.10) to $r=D$ to obtain that

$$
D \leq 2^{n} \mathrm{e}^{D^{2} / 4 t} Z+\sqrt{2 n t} .
$$

When $t=D^{2} / 8 n$, we thus conclude that

$$
D \leq 2^{n+1} \mathrm{e}^{2 n} Z
$$

The quantitative bound of the statement follows together with (3.9). The proof of the proposition is thus complete.

\section{References}

[1] Ané, C., Blachère, S., Chafaï, D., Fougères, P., Gentil, I., Malrieu, F., Roberto, C. And Scheffer, G.: Sur les inégalités de Sobolev logarithmiques. Panoramas et Synthèses 10. Société Mathématique de France, Paris, 2000.

[2] BAKRy, D.: L'hypercontractivité et son utilisation en théorie des semigroupes. Ecole d'Eté de Probabilités de St-Flour. In Lectures on probability theory (Saint-Flour, 1992), 1-114. Lecture Notes in Math. 1581. Springer, Berlin, 1994.

[3] Bakry, D.: Functional inequalities for Markov semigroups. In Probability measures on groups: recent directions and trends, 91-147. Proceedings of the CIMPA-TIFR School (2002), Tata Institute of Fundamental Research. Narosha Publishing House, New Delhi, 2006.

[4] Bakry, D., Concordet, D. And Ledoux, M.: Optimal heat kernel bounds under logarithmic Sobolev inequalities. ESAIM Probab. Statist. 1 (1995/1997), 391-407 .

[5] BAKRy, D. And Ledoux, M.: Lévy-Gromov's isoperimetric inequality for an infinite dimensional diffusion generator. Invent. Math. 123 (1996), $259-281$.

[6] BAKRY, D. AND QIAN, Z.: Harnack inequalities on a manifold with positive or negative Ricci curvature. Rev. Mat. Iberoamericana 15 (1999), 143-179.

[7] Carlen, E.: Superadditivity of Fisher's information and logarithmic Sobolev inequalities. J. Funct. Anal. 101 (1991), 194-211.

[8] Chavel, I.: Riemannian geometry $-A$ modern introduction. Cambridge Tracts in Mathematics 108. Cambridge University Press, Cambridge, 1993.

[9] Davies, E. B.: Heat kernel and spectral theory. Cambridge Tracts in Mathematics 92. Cambridge University Press, Cambridge, 1989. 
[10] Gallot, S., Hulin, D. and Lafontaine, J.: Riemannian Geometry. Second edition. Universitext. Springer-Verlag, Berlin, 1990.

[11] Gross, L.: Logarithmic Sobolev inequalities. Amer. J. Math. 97 (1975), 1061-1083.

[12] Ledoux, M.: The geometry of Markov diffusion generators. Ann. Fac. Sci. Toulouse Math. (6) 9 (2000), 305-366.

[13] Ledoux, M.: The concentration of measure phenomenon. Mathematical Surveys and Monographs 89. American Mathematical Society, Providence, RI, 2001.

[14] LI, P.: Large time behavior of the heat equation on complete manifolds with nonnegative Ricci curvature. Ann. of Math. (2) 124 (1986), 1-21.

[15] Li, P. AND YAu, S.-T.: On the parabolic kernel of the Schrödinger operator. Acta Math. 156 (1986), 153-201.

[16] Saloff-Coste, L.: Aspects of Sobolev-type inequalities. London Mathematical Society Lecture Notes Series 289. Cambridge University Press, Cambridge, 2002.

[17] Scheffer, G.: Local Poincaré inequalities in non-negative curvature and finite dimension. J. Funct. Anal. 198 (2003), 197-228.

[18] Varopoulos, N.: Small time Gaussian estimates of heat diffusion kernels, I. The semigroup technique. Bull. Sci. Math. 113 (1989), 253-277.

Recibido: 14 de julio de 2004

Dominique Bakry

Institut de Mathématiques

Université Paul-Sabatier (Toulouse III)

31062 Toulouse Cedex 9, France bakry@math.ups-tlse.fr

Michel Ledoux

Institut de Mathématiques

Université Paul-Sabatier (Toulouse III)

31062 Toulouse Cedex 9, France

ledoux@math.ups-tlse.fr 